\title{
Analysis on Human Safety Behavior Mode during the Production Process ZHOU Gang ${ }^{1,2}$, XUE Jiao', WANG Hao ${ }^{1}$, ZHANG Qi ${ }^{1}$
}

\author{
1 College of Mining and Safety Engineering, Shandong University of Science and \\ Technology, Qingdao, China 266590
}

\section{Division of Earth Science \& Resource Engineering, Commonwealth Scientific and Industrial Research Organisation, P.O. Box 883, Kenmore, Brisbane, QLD 4069, Australia}

\begin{abstract}
With the rapid development of science and technology, the ratio of accidents that caused by human unsafe behavior is getting higher and higher in complex industrial systems. Aiming at the situation of lacking the basic theory research about human safety behavior, taking the construction of overall model of human safety behavior as the purpose, analysis of human safety behavior's shaping factors as the main line, the characteristic analysis of human unsafe behavior as the basis, with the method of theoretical analysis and progression reasoning, the systematic research on the basic theory hierarchy construction of human safety behavior was carried out from the aspects of human unsafe behavior causation classification, human cognitive behavior model, human safety behavior types, which provided a theoretical basis for preventing and controlling accidents due to human factor better.
\end{abstract}

Key words: human safety behavior; causation analysis; behavior type; shaping factors; behavior pattern

\section{Introduction}

With the development of science and technology, in the complex man-machine-environment system, the reliability of equipment continues to be improved, the operating environment has been greatly improved. Due to the human characteristics in physical, psychological, social and spiritual aspects, more plastic and difficult to control, the accidents caused by human factors are getting more and more. Analysis shows that in some large catastrophic accidents, the number of casualty accidents caused by human factors directly or indirectly accounted for $70 \sim 90 \%$ of the total casualty accidents. 7500 casualty accidents since twenty-first Century in USA happened, $76 \%$ of them were caused by human errors. In Japan 10463 casualty accidents leading to more than 4 days of strikes happened in manufacturing industry in $2010,76 \%$ of the accidents were caused by human error ${ }^{[1]}$. According to China State Administration of Work Safety, in 2013, the number of all kinds of production safety accidents which happened in our nation is 363383 and 79552 deaths, in which, the number of great accidents is 85 and 1438 deaths $^{[2]}$. Through the statistical analysis about casualties condition of annual production safety accidents, the rate of accidents caused by human unsafe behavior is above $80 \%$. For example, above $90 \%$ of the death accidents are caused by the workers "three-violations" in China Huaibei Mining Group each year. In $2009,78.1 \%$ of the accident was caused by human factors in China's electric power system ${ }^{[3]}$.

Currently, the research on human 
safety behavior has gradually changed from the original form that finding what percentage human factor (human unsafe behavior) occupies in the causes of the accident and the classified analysis of human unsafe behavior into making scientific research on unsafe behavior problems in production process by constructing effective control system to unsafe behavior with complexity science principle and method.

But so far, the research on establishing the theoretical model related to human safety behavior is so poor, which makes the research about human factors in a production system less comprehensive and perfect. Therefore, it is necessary to make systematic research on the construction of human safety behavior basic theory system, which is important to make analysis and prevention on accidents scientifically and reasonably.

\section{Characteristics of human error and human unsafe behavior}

Human error refers to the behavior results deviation from the target or exceeds the acceptable boundary, and produces bad influence. According to the view of behavior psychology, human behavior mode can be expressed: $S-O-R$, that is stimulus-organism-response. The manifestation of human error lies in: error when human apperceiving the environmental information, making information stimulate the human brain; error when the brain processing information and making decision; error when behavior outputting, et $\mathrm{a}^{[4]}$. For these three aspects, Petersen attributed the human error to the three aspects of overload, decision-making error and cause of ergonomics ${ }^{[5]}$. There are three ways in the classification of human error, that is behavioristic, relational and conceptive. Human error is of the following several characteristics ${ }^{[6]}$ : (1) repeatability; (2) potentiality and irreversibility; (3) impeled by situation environment often; (4) inherent variability; (5) maintainability; human learning capacity.

However, human unsafe behavior refers to those human acts which cause or may lead to an accident, they are the direct causes of the accident. It contains two connotations: one is the behavior that is of major probability of accident (accumulated injury); and another is the behavior that goes against reducing disaster loss. Generally speaking, unsafe behavior is a kind of human error that occurs in the production process, and can directly lead to accidents, also it is a special case of human error. In the view of human psychological state, human unsafe behavior can be divided into intentional type and unintentional type.

\section{Causation analysis of human error and human unsafe behavior}

\subsection{Causation analysis of human error}

From an ergonomic point of view, human error causation can be summarized as follows: internal factors and external factors. (1) internal factors, due to the factors of operator himself uncoordinated with the machine system. (2) external factors, referring to the system design (interface, work environment, organization and management, et al) lacking a good application effect in human-machine engineering criterion, which results in hiding the factors that can easily lead to operator error, and mainly including human-computer interface, human-environment interface and human-human interface.

From a psychological perspective, possible sources of human error mainly are: feeling, perception, memory, thinking, attention, mood (emotion), will, 
character, temperament, ability, awareness, needs, motivations, interests, ideals, beliefs, values, stress (tension), fatigue, monotony, interpersonal psychology. Moreover, learning, speech and so on should also be included.

But in the view of cognitive psychology, the main causation of human error $\operatorname{are}^{[7,8]}$ : (1) information input errors, including visual failure, auditory failure, olfactory failure and so on; (2) information process errors, including inadequate training, lack of experience, the poor psychological quality, man-machine system design errors; (3) information output errors, et al.

\subsection{Causation analysis of human unsafe behavior}

\subsubsection{Causes of intentional unsafe behavior}

There are both subjective and objective reasons: (1) subjectively, the psychological factors occupy an important position, such as fluke mind, quick success; herd mentality; over confidence, trying to be brave, et al; (2) objectively, the conditions of unsafe behavior are created by the loose management and bad maneuverability of rules and regulations.

\subsubsection{Causes of unintentional unsafe behavior}

Human behavior is a kind of reaction process on the response to external stimulus, both conscious and unconscious behavioral responses can lead to unintentional unsafe behavior. (1) human internal causes, including mental, physical ,technical level aspects and so on. (2) human external causes, such as administrative reasons, educational reasons, environmental reasons, social reasons and so on, external things and circumstance changes are important predisposing factors to human unsafe behavior.

Both intentional and unintentional unsafe behavior have close relationship with human psychology .Intentional unsafe behavior is often caused by bad personality tendencies (such as, not earnest, not serious, inappropriate needs) and some bad character (such as self-willed, lazy, rude, arrogant); while, low capacity and some bad character (such as careless, cowardly, self-contemptuous, weak-minded) often leads to unintentional unsafe behavior; and good personality psychology (such as strong will) can be conducive to overcome the unsafe behavior ${ }^{[9]}$.

\section{Model of human cognitive behavior}

Human cognitive process is a complex psychological activity process, during the process, human brains reflect the characteristics and relationship of objective things, and expose their influence and effect on human. It is also a process to decipher the human thinking "black box", aiming at studying human thinking process so as to explore the mechanism of human error and explain human error behavior.

\subsection{Cognitive control modes}

Attention mode refers to a kind of psychological activities, during which human just select one or partial simulation to respond among numerous stimulation in individual situation, and obtain perceptual experience. There are two of the most important factors influencing attention: First, the individual motives or needs; secondly, the characteristics of the stimulus itself. So the cognitive process controlled by attention mode is a kind of operators' psychological characteristic when one is a beginner or is not familiar with the equipment or complete unskilled operation. The characteristic of attention 
mode in the cognitive process is "slow". It requires operators' conscious monitoring and attention as well as feedback-driven, therefore it occupies considerable proportional part in the human ability of processing information available.

Schematic mode is a basic mode, by which individual can understand the world around. Cognitive structure would be used to check, to know and understand the environment when individuals encounter to the situations of external stimulus ${ }^{[10]}$. For example, the corresponding schema in the minds is activated when a stimulus signal (for instance, the information from alarm or graphic record display) appears, and corresponding behavior is required. As schematic mode's much lower requirements in the capacity of individual information processing than attention mode, empirical errors are made easily.

\subsection{Cognitive rules}

Cognitive process, a complex mental process, is dominated by two rules ${ }^{[2,5,10]}$. The first rule is the "minimal contribution" principle, also known as "cognitive economy" principle. This principle holds that during the cognitive processes, human tend to select preferentially. Because schematic mode is used more easily and has less limitation of resources than attention mode, some scenarios can be dealt with by experience. The second is "behavior after given meaning". People often try to figure out some meanings of ambiguous data in the cognitive process, once understanding the implication of the data, then will form a strong ideological resistance to the new change of situation. This cognitive rule of "confirming prejudice" holds that "once people accept a view, a picture consistent with the view will be depicted in support of this view". This cognitive tendency of "behavior after given meaning" is often seen in nuclear power plant case study, and "confirming prejudice" is also known as "cognitive prejudice".

\subsection{Information processing modes of human}

Information exchange is the most essential link in the human production activities. Therefore the function of human must be analyzed from the perspective of information theory. The role people play in the specific operating activities of the production systems can be likened to a process of information transmission and processing. Thus starting from the mode of human safety behavior, people can be regarded as a single-channel information processing system with limited transmission capacity and a motor output system. The system is as shown in Figure 1. 


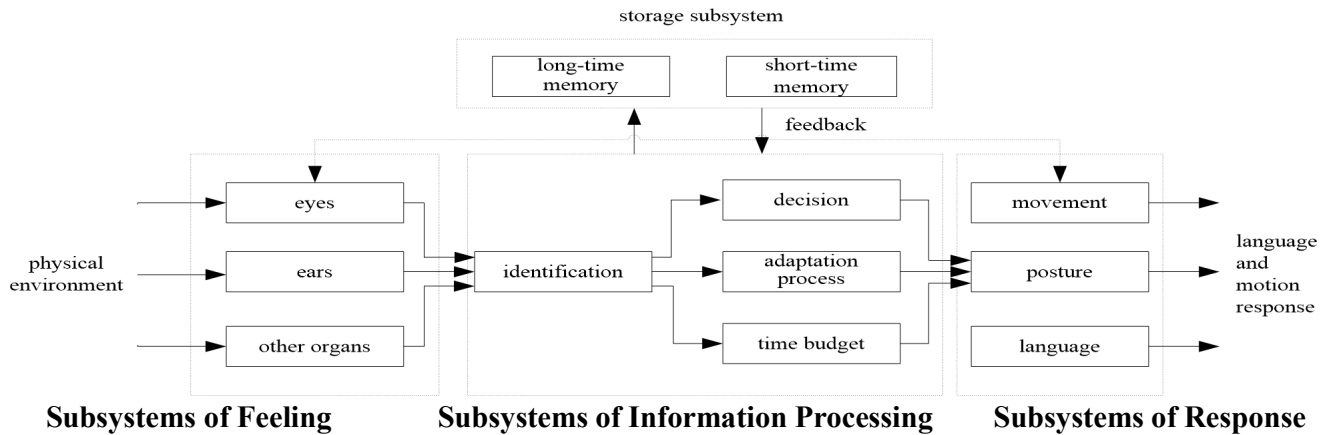

Fig.1: Information processing system and motor output system of human

In addition, Rasmussen proposed quantitative information processing model (Figure 2) step-ladder in the 1980s, which has became the basis of human error classification framework widely ${ }^{[11]}$. In this model, the human cognitive process is divided into eight stages: excitation, observation, identification, interpretation, evaluation, target selection, order selection, order execution. Its core lies in the existence of cut set among eight cognitive stages, which can reduce the amount of information processing, reflect the effectiveness and economy of human cognition, the result depends on the individual familiarity with the task. These cut sets are given by dotted line in the figure. However, because the match relationship between the current situation

\section{Mode of human safety behavior}

Rasmussen proposed three related levels of personnel performance in 1983. According to Rasmussen's three levels behavior model of SRK, the human safety behavior can be divided into three categories, and they are skill-based, rule-based and knowledge-based behavior, which represents three different human cognitive levels ${ }^{[12-14]}$.

(1) skill: referring to the very close coupling relationship between information input and human reaction, with the main feature of subconscious and experience is the key, the existence of such kind of cut set means increasing opportunities of human error.

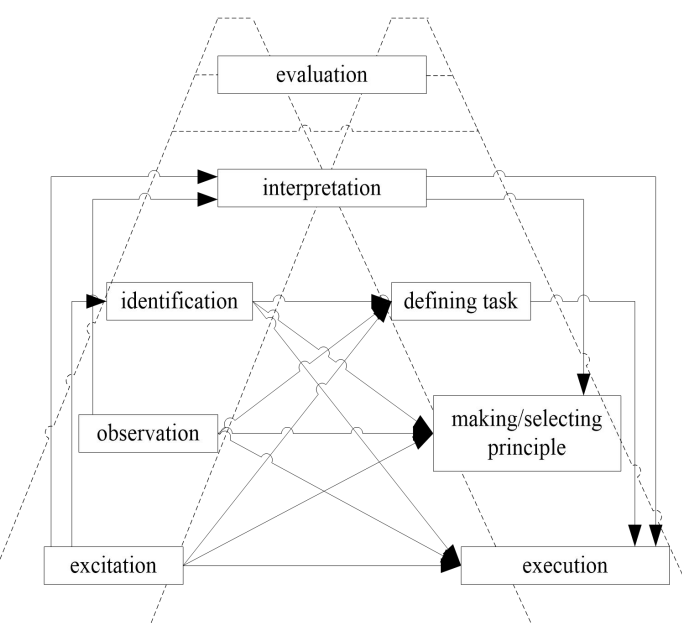

Fig.2: Ladder model of human decision

reaction and operation without interpreting the obtained information; (2) rule, the results of human behavior based on the guidance of rules or procedures directly affected by the understanding or master degree of rules/procedures; (3) knowledge, the action programs diagnosed and developed based on operators' knowledge and experience when event beyond the coverage of rules/procedures. The basic model of human cognitive safety behavior is shown in Figure 3. 


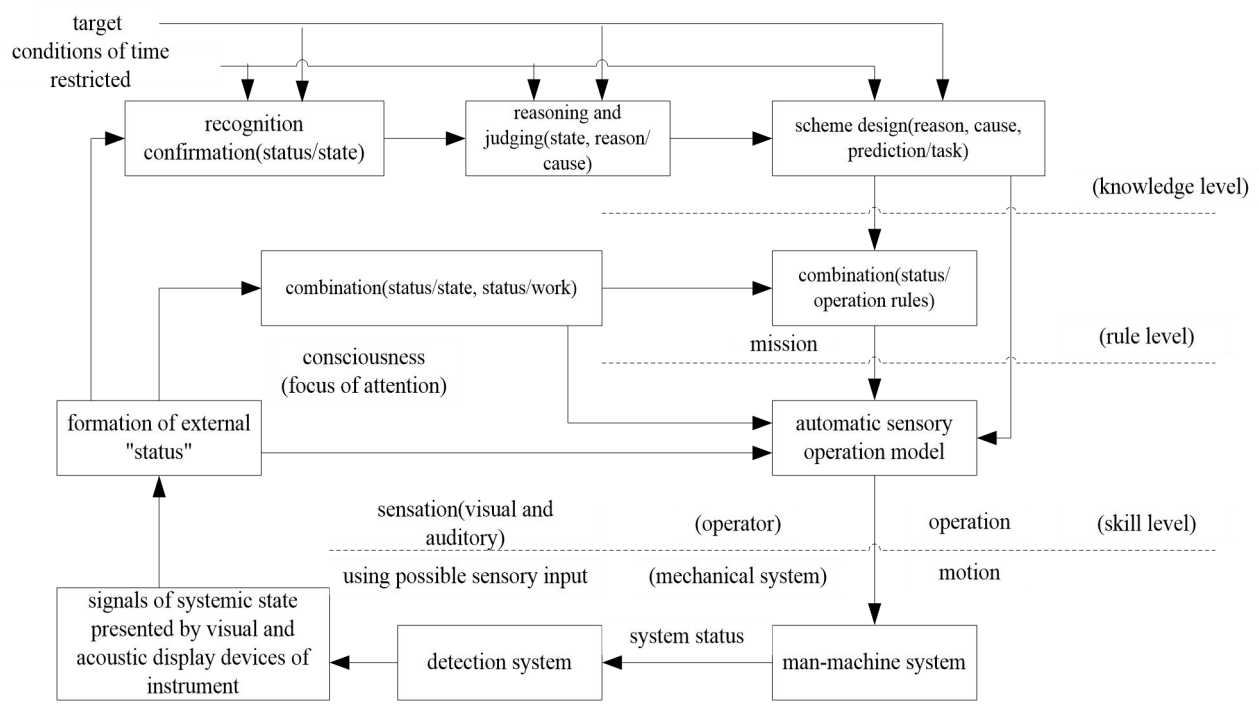

Fig.3: Graph of the basic model of human cognitive safety behavior

\section{Overall analysis on shaping factors and mode of human safety behavior} According to the causation analysis of human error and human unsafe behavior, combined with analysis results of cognitive behavior model and safety behavior mode, the shaping factors of human safety performance (PSFs) are divided into six parts: physical safety, psychological safety, safety management, engineering psychology, life important events and influence of different cultural practices, et al. Human safety behavior mode can be constructed further based on the shaping factors of human safety behavior, which is as shown in Figure 4. 


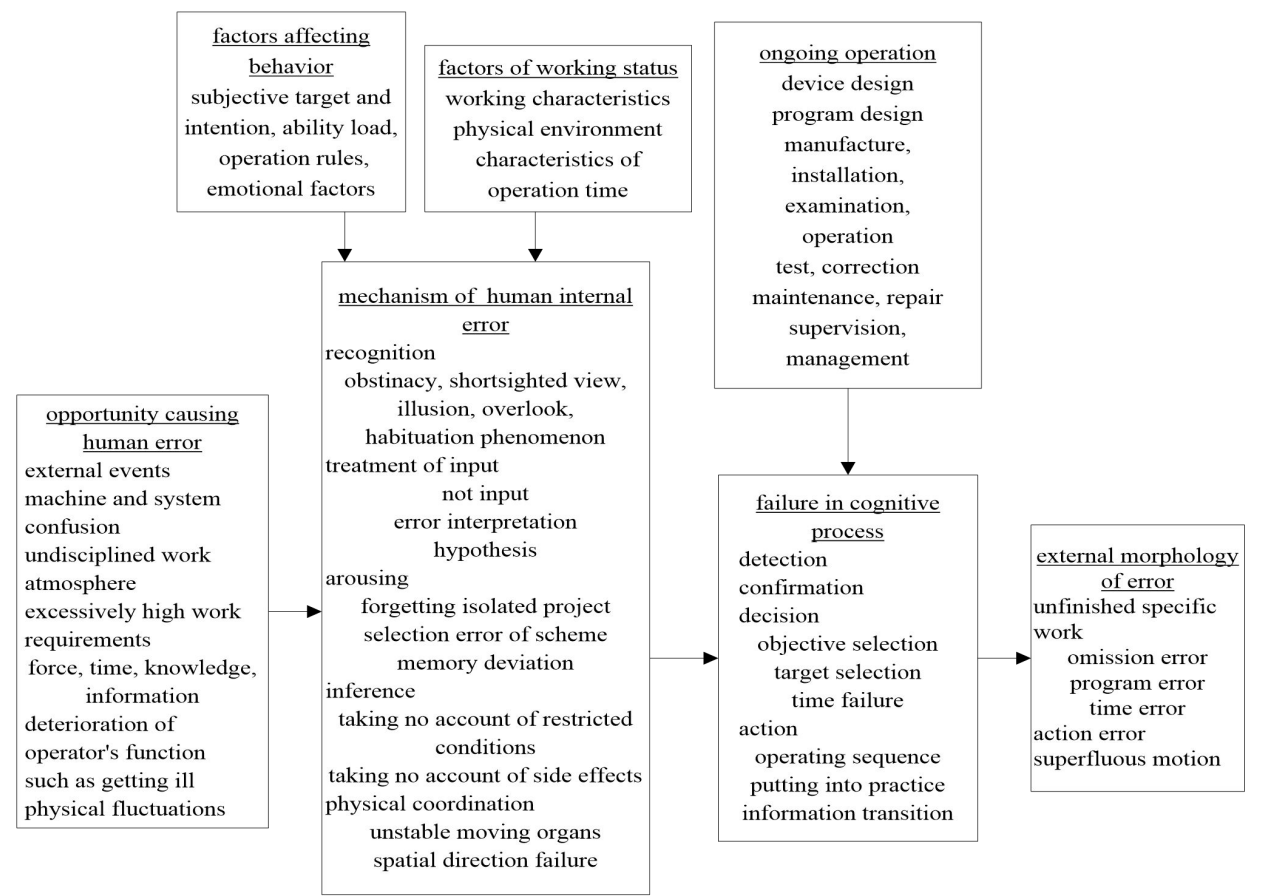

Fig.4: Overall analysis on human safety behavior mode

\section{Conclusions}

According to the disadvantages that the research results on human safety behavior is relatively decentralized, less systemic and logic, this article makes a systematical research on the construction of human safety behavior basic theory system from the aspects of human unsafe behavior causation classification, human cognitive behavior model and human safety behavior mode based on characteristics analysis of human unsafe behavior. Finally, the shaping factors of human safety behavior are formed, including physiological safety, psychological safety, safety management, engineering psychology, life important events and the influence of different cultural practices. And the overall mode of human safety behavior is constructed, which offers a direction for refining the work of preventing and controlling accidents due to human factors. But how to further perfect the overall mode of human safety behavior in practice is still the direction that the author needs to work hard in.

\section{Acknowledgments}

This work was financially supported by SDUST Research Fund, Shandong Province "Taishan Scholar" Project Fund, Collaborative Innovation Center of Coal Safe and Efficient Mining Technology and Equipment of Shandong Province, Science Research Innovative Group of College of Mining and Safety Engineering of SDUST (2012ZHTD06).

\section{References}

[1] Gang ZHOU, Jun XIE, Wei-min CHENG, et al. Evaluation method research of human safety behavior based on rough set theory[C]. Proceedings 2011 IEEE 18th International Conference on Industrial Engineering and Engineering Management, IEEE Press, 2011, 
1249-1253.

[2] State Administration of Production Safety Supervision and Management. http://media.chinasafety.gov.cn:8090/i System/shigumain.jsp, 2014.

[3] ZHOU Gang, CHENG Weimin, CHEN Lianjun. Data mining for knowledge system of miner safety behavior indexes based on genetic algorithm[C]. 2010 International Conference on Mine Hazards Prevention and Control. Amsterdam-Paris, France: Atlantis Press, 2010, 652-658.

[4] ZHOU Gang, CHENG Weimin, ZHUGE Fumin, et al. Analysis and exploration on correlative theories of man-made errors and human unsafe behaviors[J]. China Safety Science Journal, 2008, 18(3), P10-14 (Ch).

[5] CHEN Baozhi. Safety Principle(second edition)[M]. Beijing: Metallurgy Industry Press, 2002.

[6] ZHANG Li. The psychological background about human error and safety in NPP $[\mathrm{J}]$. Nuclear Power Engineering, 1992, 11(4), P91-96 (Ch).

[7] CHENG Weimin, ZHOU Gang, WANG Gang, et al. Psychological measurement and analysis on human unsafe behavior[J]. China Safety Science Journal, 2009, 19(6), P29-34 (Ch).

[8] ZHOU Gang, CHENG Weimin, WANG Gang. Application of psychometric technology to analysis for human unsafe behavior[C]. 2009 CHINA-JAPAN Symposium on Safety Management Theory and
Practice, China University of Mining and Technology Press, 2010, 197-201 (Ch).

[9] LIU Yisong. The discuss of unsafe behavior in safety management[J]. West-china Exploration Engineering, 2005, (6), P226-227 (Ch).

[10] ZHANG Li. Technology of Human Reliability Analysis in Probabilistic Safety[D]. Changsha, Hunan province: Hunan University, 2004.

[11] CHEN Lianjun, LIU Zhaoxia. Application of two quality diagnostic theory in performance evaluation of coal miner[J]. Mining \& Processing Equipment, 2005, 33(8), P139-140 (Ch).

[12] ZHOU Gang, CHENG Weimin, BIE Haiyan, et al. Safety analysis and assessment for human-machine-environment systematic engineering[C]. Progress in Safety Science and Technology: Vol VI, Beijing: Science Press/Science Press USA Inc., 2006, 552-556.

[13] GUO Yanpei, CHENG Weimin, WU Lirong, et al. Diagnosis and assessment of human unsafe behavior[C]. Progress in Safety Science and Technology: Vol VI, Beijing: Science Press/Science Press USA Inc., 2006, 613-616.

[14] Rasmussen J.. Skills, rules, knowledge, signals and symbols and other distinctions in human performance modes[J]. IEEE Transactions: Systems, Man \& Cybernetics SMC-13, 1983, 257-267. 